\title{
A Solution Framework in Traffic Congestion Management Using RFID Technology
}

\author{
Huan Wang ${ }^{\mathrm{a}}$, Jialiang $\mathrm{He}^{\mathrm{b}}$, Yu Pan
}

College of Information and Communication Engineering, Dalian Nationalities University, China

a ${ }^{*}$ peak_email@sina.com, ${ }^{\mathrm{b}}$ urchin2012@sina.com, ${ }^{\mathrm{c}}$ virtualcat@21cn.com

Keywords: RFID; Traffic congestion; Automatic Detection

\begin{abstract}
RFID is a technology which is used to identify remote objects equipped with RFID tags by wireless scanning without manual intervention; it can be used in traffic congestion management. In this paper, we propose a framework of traffic congestion management using RFID technology; a RFID tag management process for every vehicle subsequently.
\end{abstract}

\section{Introduction}

Traffic congestion is characterized by slower speeds, longer waiting times. The road networks aren't suitable for increasing vehicle volume, which leads to traffic congestion. Different technologies can detect traffic congestion and make congestion management more efficient, but these technologies have shortcomings, such as installation problems, complexity, cost, etc. Radio Frequency Identification (RFID) is a technology which is used to identify remote objects embedded with RFID tags by wireless scanning without manual intervention [1], which has been used traffic management widespread [2-4], RFID can be an easier, efficient and low-cost congestion detection technology.

A typical RFID system is composed of a backend server, readers and tags. According to power supply, RFID tag is categorized into three types as follows: Active tag; Passive tag; Semi-Active tag. Active RFID device contain a battery and can transmit signals autonomously, it does not depend on readers to initiate a communication, and it can send unique beacons to inform its presence periodically; so it is suitable for using in traffic management.

The comparison with some technologies than can be used in traffic congestion detection as follows:

(1)Inductive loop detection

It can be placed in a roadbed to detect vehicles by measuring the vehicle's magnetic field; the simplest detectors simply count the number of vehicles during a unit of time; loops can be placed in a single lane or across multiple lanes. However, it has some shortcomings: the error rate is quite high; maintenance is very tedious; traffic cannot be managed locally.

(2)Video data analysis

Video comes from the cameras; the built-in software gets information from that video; information then fed into the fuzzy system that outputs the level of traffic congestion. However, it has two shortcomings: the overall system is quite expensive; the fuzzy algorithm is not very accurate.

(3)Passive infrared sensors

Passive sensors detect energy emitted from vehicles, road surfaces, and other objects in their field of view and by the atmosphere; the captured energy is focused by an optical system onto an infrared-sensitive material which converts the reflected energy into electrical signals; real-time signal processor analyze the signals to detect presence of a vehicle.

(4)Wireless sensor network

Magnetic sensors are deployed by the road intersection to detect vehicles; the sensors send the collected data to the Data Center (DC); DC process the data and dynamically controlled the traffic light; a high vehicle density in a particular lane causes a traffic signal in that particular direction to remain open for larger duration thus adaptively controlling the signal. 
(5)Active RFID

No direct line of sight required for identification and enables very specific detection of vehicles; simultaneous multiple detection of vehicles are possible by using RFID.

In Singapore, public transport buses and trains employing RFID cards is well known. Traffic into crowded downtown areas is regulated by variable tolls imposed using an active tag system combined with the use of stored-value cards. Congestion pricing is charged for the vehicles that enter central districts in the prime time, the vehicles owners would pay different fees according to various time and vehicle types. The implementation of this scheme as follows:


Figure.1 Traffic toll system in Singapore

\section{The framework of vehicles congestion management}

The framework consisting of the following components:

(1)Active RFID tag has been installed in each vehicle unit.

(2)Roadside RFID Reader units that would capture information from vehicles and forward them to control station using GSM/GPRS network services.

(3)Control Station for accepting vehicle data, doing necessary computations based on predefined traffic-rules, issuing traffic-management messages to the relevant vehicles and computing necessary billing for each vehicle. 


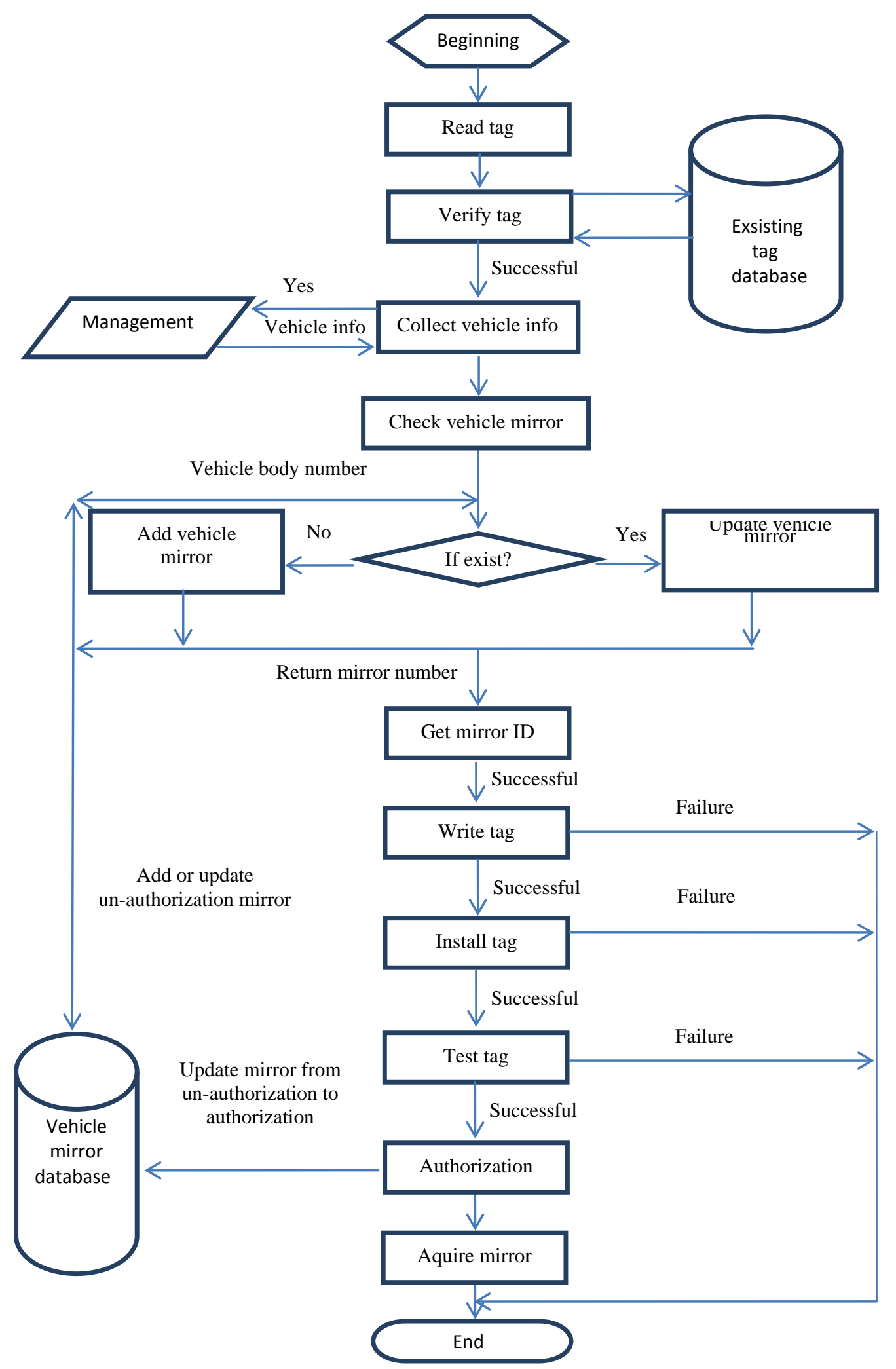

Figure.2 RFID tag management process for each vehicle[4]

Another important pre-requisite for this information system is governance and legislation. With legal support, make it mandatory to fit RFID-enabled devices in the cars. Built-in RFID tag management process refers to Figure.2. 


\section{Conclusion}

RFID can be used in traffic management widespread. In this paper, we propose a framework of traffic congestion management using RFID technology; a RFID tag management process for every vehicle subsequently. Traffic management concerns with everyone in the world, it is an important research object and has attracted many searchers to embark on it.

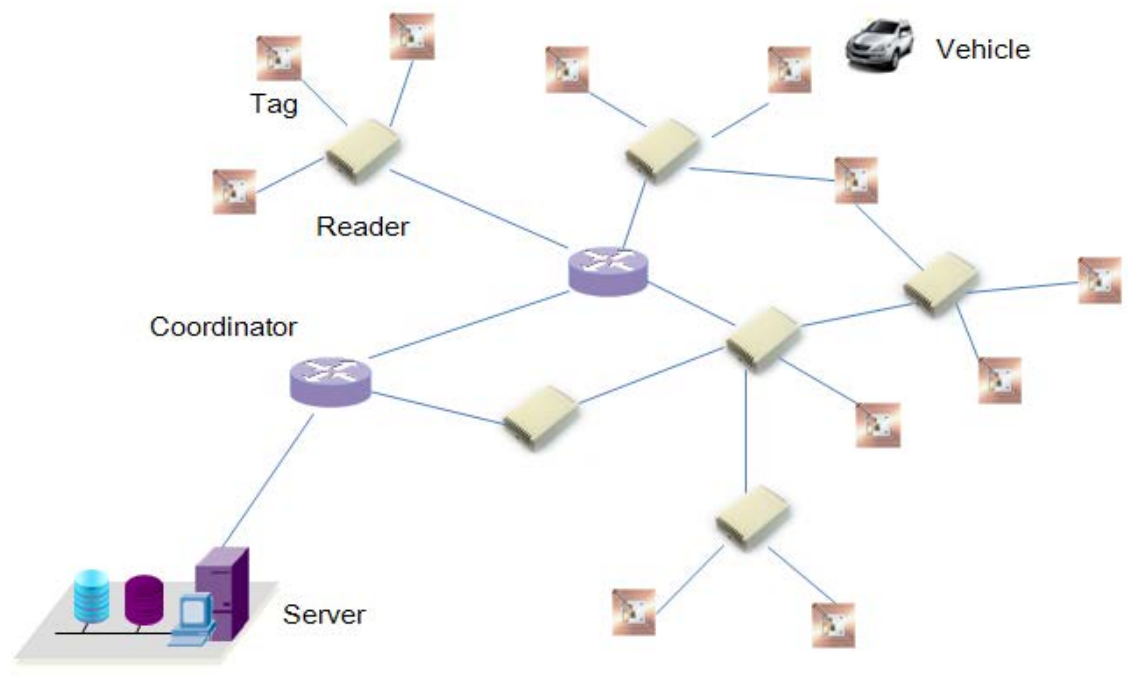

Figure.3 Solution Framework

\section{Acknowledgements}

This work was supported by "National Undergraduate Training Programs for Innovation and Entrepreneurship of Dalian Nationalities University”.

\section{References}

[1] He Jialiang, Ouyang Dantong, Ye Yuxin, “An Efficient Lightweight RFID Authentication Protocol for Low-cost Tags”, Advances in Information Sciences and Service Sciences, vol.3, no.9, pp.331-338, (2011).

[2] He Xu, Suoping Wang, Ruchuan Wang, “A Novel RFID Reader System Framework based on Peer-to-Peer Network”, International Journal of Advancements in Computing Technology, vol.3, no.3, pp.104-110,(2011).

[3] L. Yan, Y. Zhang, L.T. Yang, H.S. Ning, “The Internet of Things: From RFID to the Next-Generation Pervasive Networked Systems”, Wireless Networks and Mobile Communications, Auerbach Publicatios, (2008).

[4] He Jialiang, Shen Peng, Xu Zhiqiang. "Inspection Solution of Unlawfully Modified Vehicle by Sensor-RFID Technology”. Sensors and Transducers Journal, In press, (2013)

[5] Feng Haiyang, He Jialiang, Zhang Lijun, Meng Fangang, “An Application of RFID Technology in Traffic Congestion Management”, 2nd International Conference on Robotics and Electromechanical Engineering(BFM'13), In press, (2013). 